\title{
Erratum to: Using Improved Product-Coded Modulation Codes to Reduce the Peak-to-Average Power Ratio in Single-Carrier Frequency Division Multiple Access Systems
}

\author{
Hsin-Ying Liang ${ }^{1}$ Cheng-Ying Yang ${ }^{2}$ Kuang-Hao Lin ${ }^{3}$. \\ Chia-Hsin Cheng ${ }^{3}$
}

Published online: 29 August 2016

(C) Springer Science+Business Media New York 2016

\section{Erratum to: Wireless Pers Commun DOI 10.1007/s11277-016-3532-1}

In the initial, online publication, the last author's affiliation, photograph, and biography were incorrect. The online article has been updated to correct the errors.

The online version of the original article can be found under doi:10.1007/s11277-016-3532-1.

Hsin-Ying Liang

hyliang@gm.cyut.edu.tw

1 Department of Information and Communication Engineering, Chaoyang University of Technology, Taichung, Taiwan

2 Department of Computer Science, University of Taipei, Taipei, Taiwan

3 Department of Electrical Engineering, National Formosa University, Yunlin, Taiwan 